\title{
Due Date Single Machine Scheduling Problems with Nonlinear Deterioration and Learning Effects and Past Sequence Dependent Setup Times
}

\author{
Hüseyin Ceylan \\ Kırıkkale Vocational School, Kırıkkale University, 71451 Kırıkkale, Turkey \\ Correspondence should be addressed to Hüseyin Ceylan; husceylan@hotmail.com
}

Received 26 February 2014; Revised 7 July 2014; Accepted 9 July 2014; Published 23 July 2014

Academic Editor: Sabri Arik

Copyright (C) 2014 Hüseyin Ceylan. This is an open access article distributed under the Creative Commons Attribution License, which permits unrestricted use, distribution, and reproduction in any medium, provided the original work is properly cited.

We present some problems against due dates with nonlinear learning and deterioration effects and past sequence dependent setup times. In this study, two effects (learning and deterioration) are used for the same processing time. The processing time of a job is shorter if it is scheduled later, rather than in the sequence. This phenomenon is known in the literature as a "learning effect." On the other hand, in many realistic scheduling settings, a job processed later consumes more time than the same job processed earlierthis is known as scheduling with deteriorating jobs. In the past sequence dependent setup times approach, the setup time of a job is proportionate to the sum of processing times of the jobs already scheduled. In this study, we demonstrated that some problems with due dates remain polynomially solvable. However, for some other problems, we concentrated on finding polynomially solves under their special cases.

\section{Introduction}

This paper addresses several problems against due dates with nonlinear learning and deterioration effects and past sequence dependent setup times. A scheduling problem is very important for a manufacturing system. So, numerous scheduling problems have been studied by researchers. In classical scheduling theory, the processing times of jobs are assumed to be known and fixed. However, in many realistic settings, workstations improve continuously as a result of repeating the same or similar activities such as the production of glass crafts by a skilled craftsman [1], the car repair or maintenance by a worker, and the patient diagnosis and treatment by a doctor [2]. Thus, the processing time of a job is shorter if it is scheduled later, rather than in the sequence. Mosheiov [3] determined that this phenomenon is known in the literature as a "learning effect." Biskup [4] was the first to investigate the learning effect in the scheduling problems. $\mathrm{He}$ assumed that the production time as a function of the number of repetitions of the production of a single item with a learning effect decreases. A number of researchers [35] studied E/T problem with learning effect, which is with a single machine and no deterioration effect. They solved unrestricted common due date problem as an assignment problem. In this work, we used Biskup's learning effect (see [4]). Consider

$$
p^{*}=p_{i} \times r^{a},
$$

where $p^{*}$ is basic processing time of job $i$ performed at position $r$ when $a \leq 0$ is learning index.

There is a growing interest in the literature to study scheduling problems of deterioration jobs; that is, jobs whose processing times are increasing functions of their starting times. Mosheiov [6] pointed out another case searching for an object under worsening weather or growing darkness. Although J. N. D. Gupta and S. K. Gupta [7] provided an example of steel rolling miles where the temperate of an ingot, while waiting to enter the rolling machine, drops below a certain level, which required the ingot to be reheated before rolling, Kunnathur and Gupta [8] gave a fire fighting example where the time and effort required to control a fire increase if there is a delay in the start of the fire-fighting effort. Alidaee and Womer [9] classified deteriorating jobs models into three 
different types: linear, piecewise linear, and nonlinear. In this paper, we are interested in nonlinear deterioration effect. One has

$$
P_{r}(t)=\left(p_{r}+\alpha t_{r}^{b}\right)
$$

where $t_{i}$ is starting time of $J_{i}$ and $\alpha(\alpha>0)$ and $(b>0)$ are nonlinear deterioration effect, which is the amount of increase in the processing time of a job per unit delay in its starting time.

In the scheduling literature, many researchers have worked different combinations of these three special cases to solve single machine, parallel machine, and flow shop scheduling problems. In this paper, we introduce firstly position dependent learning effect, nonlinear deterioration effect, and past sequence dependent setup time to solve due date problems in the single machine scheduling environment.

In the literature, there are a few studies on scheduling problems with effects of learning and deterioration simultaneously. Wang and Cheng [10] studied single machine scheduling problem with deteriorating jobs and learning effects to minimize the makespan when Wang [11] solved polynomially single machine scheduling problems with deteriorating jobs and learning effects. Toksari and Güner [12] proposed mixed nonlinear integer model for parallel machine earliness/tardiness scheduling problem with sequence dependent setup time and the effects of learning and deterioration. In another paper, they [13] proposed that the optimum solution of the same problem is $\mathrm{V}$-shaped. Toksari and Güner [12] consider a parallel machine earliness/tardiness (ET) scheduling problem with different penalties under the effects of position based learning and linear and nonlinear deteriorations. The same authors [13] also worked on some scheduling problems with the nonlinear effects of learning and deterioration. Cheng et al. [1] derived polynomial-time optimal solutions for some scheduling problems with deteriorating jobs and learning effect. Linear deterioration effect was used in all studies on scheduling problems effects of learning and deterioration simultaneously. Güner and Toksari [14] studied the same scheduling problems with position based learning effect and linear deterioration simultaneously. We extended this study using nonlinear deterioration effect for the same problems.

\section{Problem Formulation}

Güner and Toksari [14] considered the position dependent learning effect of a job and linear deterioration effect for the same processing time. In this study, these two effects are combined as follows:

$$
p^{*}=\left(p_{r}+\left(\alpha \times t_{r}^{b}\right)\right) r^{a}
$$

There are $n$ jobs to be scheduled on single machine. If job $i, i=$ $1,2, \ldots, n$, is being scheduled in position $r$ in a sequence, its actual processing time is $p^{*} \cdot p_{r}$ is basic processing time of job scheduled in position $r . \alpha(\alpha>0)$ and $(b>0)$ are nonlinear deterioration effect, which is the amount of increase in the processing time of a job per unit delay in its starting time. $a(a<0)$ is the learning index. $t_{r}$ is starting time of job scheduling in position $r$, and $\left(C_{r-1}^{*}\right)$ is the actual completion time of the job scheduled in position $(r-1)$. Thus, the actual processing time $p^{*}$ is formulized as follows:

$$
p^{*}=p_{r}+\left(\alpha \times\left(C_{r-1}^{* b}\right)\right) r^{a},
$$

where $\left(C_{r-1}^{*}\right)$ is the actual completion time of the job scheduled in position $(r-1)$.

We also take setup times into consideration in the scheduling model by adopting the notion of Koulamas and Kyparisis [15] that setup times are past sequence dependent. Koulamas and Kyparisis [15] motivate the assumption of $\mathrm{p}$ s-d setup times by the manufacturing of integrated circuit (IC) boards. Specifically, an IC board consists of a number of electronic components mounted on it and the processing of any electronic component will have an adverse effect on the "readiness" of other components on the board due to the passage of electricity through the board. Consequently, each component prior to processing requires a setup operation to restore it to "full-readiness" status and the setup time depends on the component's degree of "unreadiness," which is proportional to the actual processing times of the already processed components. Thus, the $\mathrm{p}$-s-d setup time $\left(s_{r}^{\mathrm{psd}}\right)$ of job $j$ if it is scheduled in the $r$ th position in a sequence is given as follows:

$$
s_{1}^{\mathrm{psd}}=0, \quad s_{r}^{\mathrm{psd}}=\gamma \sum_{i=1}^{r-1} p^{*}
$$

where $\gamma \geq 0$ is a normalizing constant. The value of the normalizing constant $\gamma$ determines the actual lengths of the required setups and, when $\gamma=0$, there is no need for any p-s-d setups (see [15]). We denote the effects of the learning and deterioration in (3) by learning effect and nonlinear deterioration effect and denote the $\mathrm{p}$-s- $\mathrm{d}$ setup given in (5) by $s_{\mathrm{p}-\mathrm{q}-\mathrm{d}}$.

\section{Some Single Machine Scheduling Problems with Individual Due Dates under Nonlinear Effects of Learning and Deterioration}

Before presenting the main results, we first present a lemma, which will be used in the proofs of the theorems in the sequel.

Lemma 1. Consider

$$
\begin{aligned}
C^{*}=[ & {\left[M+p_{1}+\left(\alpha \times M^{b}\right)\right] } \\
& +\sum_{i=2}^{r}\left[\left[p_{i}+\left(\alpha \times\left(C_{r-1}^{* b}\right)\right)\right](i)^{a}\right] \\
& \left.+\gamma \sum_{i=1}^{r-1} \sum_{j=1}^{i}\left[\left[p_{j}+\left(\alpha \times\left(C_{r-1}^{* b}\right)\right)\right](j)^{a}\right]\right]
\end{aligned}
$$


where

$$
\begin{gathered}
p^{*}=\left[p_{r}+\left(\alpha \times\left(C_{r-1}^{* b}\right)\right)\right](r)^{a}, \\
C_{1}^{*}=s_{1}^{*}+p_{1}^{*}=p_{1}+M+\left(\alpha \times M^{b}\right),
\end{gathered}
$$

when $M$ is starting time of the schedule.

Proof. One has

$$
\begin{gathered}
s_{1}^{*}=\gamma(0)=0 \\
p_{1}^{*}=\left[p_{1}+\left(\alpha \times M^{b}\right)\right](1)^{a}=p_{1}+\left(\alpha \times M^{b}\right) \\
C_{1}^{*}=s_{1}^{*}+p_{1}^{*}=M+p_{1}+\left(\alpha \times M^{b}\right) \\
s_{2}^{*}=\gamma\left(p_{1}^{*}\right) \\
C_{2}^{*}=\left[C_{1}^{*}+s_{2}^{*}+p_{2}^{*}=M+\left[\left[p_{1}+\left(\alpha \times M^{b}\right)\right](1)^{a}\right]\right. \\
\left.+\left[\left[p_{2}+\left(\alpha \times\left(C_{1}^{* b}\right)\right)\right](2)^{a}\right]+\gamma\left(p_{1}^{*}\right)\right] \\
s_{3}^{*}=\gamma\left(p_{1}^{*}+p_{2}^{*}\right) \\
p_{3}^{*}=\left[p_{3}+\left(\alpha \times\left(C_{2}^{* b}\right)\right)\right](3)^{a}, \\
C_{3}^{*}=C_{2}^{*}+s_{3}^{*}+p_{3}^{*} \\
=M_{+}+\left[\left[\left[p_{1}+\left(\alpha \times M^{b}\right)\right](1)^{a}\right]\right. \\
+\left[\left[p_{2}+\left(\alpha \times\left(C_{1}^{* b}\right)\right)\right](2)^{a}\right] \\
+\left[\left[p_{3}+\left(\alpha \times\left(C_{2}^{* b}\right)\right)\right](3)^{a}\right] \\
\left.+\gamma\left(p_{1}^{*}+p_{2}^{*}\right)\right]
\end{gathered}
$$

then, we can obtain the following:

$$
\begin{gathered}
p^{*}=\left[p_{r}+\left(\alpha \times\left(C_{r-1}^{* b}\right)\right)\right](r)^{a} \\
C^{*}=\left[\left[M+p_{1}+\left(\alpha \times M^{b}\right)\right]\right. \\
+\sum_{i=2}^{r}\left[\left[p_{i}+\left(\alpha \times\left(C_{r-1}^{* b}\right)\right)\right](i)^{a}\right] \\
\left.+\gamma \sum_{i=1}^{r-1} \sum_{j=1}^{i}\left[\left[p_{j}+\left(\alpha \times\left(C_{r-1}^{* b}\right)\right)\right](j)^{a}\right]\right]
\end{gathered}
$$

where

$$
C_{1}^{*}=s_{1}^{*}+p_{1}^{*}=p_{1}+M+\left(\alpha \times M^{b}\right) .
$$

This completes the proof.

Let $\sum L_{j}, L_{\max }=\max \left\{d_{j}-C_{j} \mid j=1,2, \ldots, n\right\}, \sum T_{j}$, and $T_{\max }=\max \left\{\left\{d_{j}-C_{j} \mid j=1,2, \ldots, n\right\}, 0\right\}$ represent the total lateness, total tardiness, maximum lateness, and maximum tardiness of a given permutation, respectively. These problems with past sequence dependent setup times were studied by Biskup and Herrmann [16] and Güner and Toksari [14] studied the same scheduling problems with position based learning effect and linear deterioration simultaneously. We used the same approach with Güner and Toksari [14] to prove the theorems.

Theorem 2. The problem can be solved optimally by sequencing jobs in nondecreasing order of their processing times (SPT rule).

Proof. Consider an optimal schedule $\pi$. Assume, under $\pi$, there are two adjacent jobs, job $J_{u}$, whose sequence is $r$, followed by job $J_{v}$, whose sequence is $r+1$, such that $p_{u}<p_{v}$. $C_{u}^{*}$ and $C_{v}^{*}$ express completion times of $J_{u}$ and $J_{v}$ scheduled at positions $u$ and $v(v=u+1)$ under the nonlinear deterioration and learning effect, respectively. $M$ is starting time of the schedule.

Lateness of job in position $r$ can be calculated by

$$
L_{r}^{*}=\left(C_{r}^{*}-d_{r}\right) ;
$$

then, from Lemma 1 ,

$$
\begin{aligned}
L_{u}^{*}(\pi)= & {\left[\left[M+p_{1}+\left(\alpha \times M^{b}\right)\right]\right.} \\
& +\sum_{i=2}^{r-1}\left[\left[p_{i}+\left(\alpha \times C_{i-1}^{* b}\right)\right](i)^{a}\right] \\
& +\left[\left[p_{u}+\left(\alpha \times C_{r-1}^{* b}\right)\right](r)^{a}\right] \\
& \left.+\gamma \sum_{i=1}^{r-1} \sum_{j=1}^{i}\left[\left[p_{j}+\left(\alpha \times C_{j-1}^{* b}\right)\right](j)^{a}\right]\right]-d_{u},
\end{aligned}
$$

$$
\begin{aligned}
L_{v}^{*}(\pi)= & {\left[\begin{array}{l}
{[} \\
\end{array} \quad p_{1}+\left(\alpha \times M^{b}\right)\right] } \\
& +\sum_{i=2}^{r-1}\left[\left[p_{i}+\left(\alpha \times C_{i-1}^{* b}\right)\right](i)^{a}\right] \\
+ & {\left[\left[p_{u}+\left(\alpha \times C_{r-1}^{* b}\right)\right](r)^{a}\right] } \\
& \left.+\gamma \sum_{i=1}^{r-1} \sum_{j=1}^{i}\left[\left[p_{j}+\left(\alpha \times C_{j-1}^{* b}\right)\right](j)^{a}\right]\right] \\
+ & {\left[\left[\left[p_{v}+\left(\alpha \times C_{u}^{* b}(\pi)\right)\right](r+1)^{a}\right]\right] } \\
& +\gamma \sum_{i=1}^{r-1}\left[\left[p_{i}+\left(\alpha \times C_{i-1}^{* b}\right)\right](i)^{a}\right] \\
& \left.+\gamma\left[\left[p_{u}+\left(\alpha \times C_{r-1}^{* b}\right)\right](r)^{a}\right]\right]-d_{v},
\end{aligned}
$$


where $\left(C_{u}^{b *}(\pi)\right)^{r}$ explains the completion time of $J_{u}$ when it is in position $r$ at schedule $\pi$. If we assume that $t$ is total lateness up to position $r$, then

$$
\begin{aligned}
& \sum L(\pi)=t+2\left[\left[M+p_{1}+\left(\alpha \times M^{b}\right)\right]\right. \\
& +\sum_{i=2}^{r-1}\left[\left[p_{i}+\left(\alpha \times C_{i-1}^{b *}\right)\right](i)^{a}\right] \\
& +\left[\left[p_{u}+\left(\alpha \times C_{r-1}^{b *}\right)\right](r)^{a}\right] \\
& \left.+\gamma \sum_{i=1}^{r-1} \sum_{j=1}^{i}\left[\left[p_{j}+\left(\alpha \times C_{j-1}^{b *}\right)\right](j)^{a}\right]\right] \\
& +\left[\left[\left[\left[p_{v}+\left(\alpha \times C_{u}^{* b}(\pi)\right)\right](r+1)^{a}\right]\right]\right. \\
& +\gamma \sum_{i=1}^{r-1}\left[\left[p_{i}+\left(\alpha \times C_{i-1}^{* b}\right)\right](i)^{a}\right] \\
& \left.+\gamma\left[\left[p_{u}+\left(\alpha \times C_{r-1}^{* b}\right)\right](r)^{a}\right]\right]-d_{v}-d_{u} .
\end{aligned}
$$

Performing a pairwise interchange on jobs $J_{u}$ and $J_{v}$, we obtain schedule $\pi^{\prime}$. The completion times of the jobs processed before jobs $J_{u}$ and $J_{v}$ are not affected by interchange; then,

$$
\begin{aligned}
L_{v}^{*}\left(\pi^{\prime}\right)= & {\left[\left[M+p_{1}+\left(\alpha \times M^{b}\right)\right]\right.} \\
& +\sum_{i=2}^{r-1}\left[\left[p_{i}+\left(\alpha \times C_{i-1}^{* b}\right)\right](i)^{a}\right] \\
& +\left[\left[p_{v}+\left(\alpha \times C_{r-1}^{* b}\right)\right](r)^{a}\right] \\
& \left.+\gamma \sum_{i=1}^{r-1} \sum_{j=1}^{i}\left[\left[p_{j}+\left(\alpha \times C_{j-1}^{* b}\right)\right](j)^{a}\right]\right]-d_{v}, \\
L_{u}^{*}\left(\pi^{\prime}\right)= & {\left[\left[M+p_{1}+\left(\alpha \times M^{b}\right)\right]\right.} \\
& +\sum_{i=2}^{r-1}\left[\left[p_{i}+\left(\alpha \times C_{i-1}^{* b}\right)\right](i)^{a}\right] \\
& +\left[\left[p_{v}+\left(\alpha \times C_{r-1}^{* b}\right)\right](r)^{a}\right] \\
& \left.+\gamma \sum_{i=1}^{r-1} \sum_{j=1}^{i}\left[\left[p_{j}+\left(\alpha \times C_{j-1}^{* b}\right)\right](j)^{a}\right]\right]
\end{aligned}
$$

$$
\begin{aligned}
& +\left[\left[\left[\left[p_{u}+\left(\alpha \times C_{u}^{* b}(\pi)\right)\right](r+1)^{a}\right]\right]\right. \\
& +\gamma \sum_{i=1}^{r-1}\left[\left[p_{i}+\left(\alpha \times C_{i-1}^{* b}\right)\right](i)^{a}\right] \\
& \left.+\gamma\left[\left[p_{v}+\left(\alpha \times C_{r-1}^{* b}\right)\right](r)^{a}\right]\right]-d_{u} .
\end{aligned}
$$

If we assume that $t$ is total lateness up to position $r$, then

$$
\begin{gathered}
\sum L\left(\pi^{\prime}\right)=t+2\left[\left[M+p_{1}+\left(\alpha \times M^{b}\right)\right]\right. \\
+\sum_{i=2}^{r-1}\left[\left[p_{i}+\left(\alpha \times C_{i-1}^{b *}\right)\right](i)^{a}\right] \\
+\left[\left[p_{u}+\left(\alpha \times C_{r-1}^{b *}\right)\right](r)^{a}\right] \\
\left.+\gamma \sum_{i=1}^{r-1} \sum_{j=1}^{i}\left[\left[p_{j}+\left(\alpha \times C_{j-1}^{b *}\right)\right](j)^{a}\right]\right] \\
+\left[\left[\left[\left[p_{u}+\left(\alpha \times C_{u}^{* b}(\pi)\right)\right](r+1)^{a}\right]\right]\right. \\
+\gamma \sum_{i=1}^{r-1}\left[\left[p_{i}+\left(\alpha \times C_{i-1}^{* b}\right)\right](i)^{a}\right] \\
\left.+\gamma\left[\left[p_{v}+\left(\alpha \times C_{r-1}^{* b}\right)\right](r)^{a}\right]\right]-d_{v}-d_{u} .
\end{gathered}
$$

The difference between the values of $\sum L\left(\pi^{\prime}\right)$ and $\sum L(\pi)$ is

$$
\begin{aligned}
& \left(\sum L\left(\pi^{\prime}\right)-\sum L(\pi)\right) \\
& =\left[\left((\gamma+2)\left(p_{v}-p_{u}\right)(r)^{a}\right)-\left(\left(p_{v}-p_{u}\right)(r+1)^{a}\right)\right. \\
& \left.\quad+\left(\alpha\left(C_{r}^{b *}\left(\pi^{\prime}\right)-C_{u}^{b *}(\pi)\right)(r+1)^{a}\right)\right] \\
& =\left[\left((\gamma+2)\left(p_{v}-p_{u}\right)(r)^{a}\right)-\left(\left(p_{v}-p_{u}\right)(r+1)^{a}\right)\right. \\
& \left.\quad+\left(\alpha\left(p_{v}-p_{u}\right)(r)^{a}(r+1)^{a}\right)\right],
\end{aligned}
$$

$\sum L\left(\pi^{\prime}\right)-\sum L(\pi) \geq 0$, where $p_{u}<p_{v},(a<0),(b>0)$, and $(\alpha>0)$.

Consequently, $\sum L\left(\pi^{\prime}\right)>\sum L(\pi)$.

The total lateness under $\pi$ is strictly less than that under $\pi^{\prime}$. This completes the proof.

Example 3. Let $p_{1}=100, p_{2}=90, d_{1}=101, d_{2}=102, a=$ -0.322 when learning rate $\% 80, \alpha=0.1, b=0.2$ and $\gamma=0.2$. Then, sequence $\pi(S=(1,2))$ leads to $p_{1}^{*}(\pi)=100, s_{1}^{*}(\pi)=0$, $C_{1}^{*}(\pi)=100, p_{2}^{*}(\pi)=73.26, s_{2}^{*}(\pi)=20, C_{2}^{*}(\pi)=193.26$, 
and $\sum_{i=1}^{2} L_{i}(\pi)=91.26$. The SPT sequence $\pi^{\prime}(S=(2,1))$ leads to $p_{1}^{*}\left(\pi^{\prime}\right)=90, s_{1}^{*}\left(\pi^{\prime}\right)=0, C_{1}^{*}\left(\pi^{\prime}\right)=90, p_{2}^{*}\left(\pi^{\prime}\right)=81.24$, $s_{2}^{*}\left(\pi^{\prime}\right)=18, C_{2}^{*}(\pi)=189.24$, and $\sum_{i=1}^{2} L_{i}\left(\pi^{\prime}\right)=88.24$.

Theorem 4. The problem $1\left|\left[p_{r}+\left(\alpha \times t_{r}^{b}\right)\right](r)^{a}+s_{r}^{* p s d}\right| \sum T$ can be solved optimally by sequencing jobs in nondecreasing order of their processing times (SPT rule).

Proof. Tardiness $T$ is defined as $T=\{0, L\}$. The results of Theorem 2 can be transferred directly to the case of $1 \mid\left[p_{r}+\right.$ $\left.\left(\alpha \times t_{r}^{b}\right)\right](r)^{a}+s_{r}^{* \operatorname{psd}} \mid \sum T$. This completes the proof.

Theorem 5. The problem both $1\left|\left[p_{r}+\left(\alpha \times t_{r}^{b}\right)\right](r)^{a}+s_{r}^{* p s d}\right|$ $L_{\max }$ and $1\left|\left[p_{r}+\left(\alpha \times t_{r}^{b}\right)\right](r)^{a}+s_{r}^{* p s d}\right| T_{\max }$ can be solved optimally by sequencing jobs in nondecreasing order of their processing times (SPT rule) (or equivalently earliness due date (EDD) rule) if the due dates and the processing times are agreeable; that is, if $p_{u} \leq p_{v}$ implies $d_{u} \leq d_{v}$.

Proof. We would not duplicate $L_{u}^{*}(\pi), L_{v}^{*}(\pi), L_{u}^{*}\left(\pi^{\prime}\right)$, and $L_{v}^{*}\left(\pi^{\prime}\right)$ due to knowing them from Theorem 4 .

The difference between the values of $L_{u}^{*}(\pi)$ and $L_{v}^{*}(\pi)$ is

$$
\begin{aligned}
& \left(L_{v}^{*}(\pi)-L_{u}^{*}(\pi)\right) \\
& =+\left[\left[\left[\left[p_{v}+\left(\alpha \times C_{u}^{* b}(\pi)\right)\right](r+1)^{a}\right]\right]\right. \\
& \quad+\gamma \sum_{i=1}^{r-1}\left[\left[p_{i}+\left(\alpha \times C_{i-1}^{* b}\right)\right](i)^{a}\right] \\
& \left.\quad+\gamma\left[\left[p_{u}+\left(\alpha \times C_{r-1}^{* b}\right)\right](r)^{a}\right]\right]-\left[d_{v}-d_{u}\right],
\end{aligned}
$$

$L_{v}^{*}(\pi)-L_{u}^{*}(\pi) \geq 0$, where $p_{u} \leq p_{v}, d_{u} \leq d_{v},(a<0)$, and $(\alpha, b>0)$.

Consequently, $L_{v}^{*}(\pi) \geq L_{u}^{*}(\pi)$.

The difference between the values of $L_{u}^{*}\left(\pi^{\prime}\right)$ and $L_{v}^{*}(\pi)$ is

$$
\begin{aligned}
& \left(L_{u}^{*}\left(\pi^{\prime}\right)-L_{v}^{*}(\pi)\right) \\
& \quad=\left[\left((\gamma+1)\left(p_{v}-p_{u}\right)(r)^{a}\right)-\left(\left(p_{v}-p_{u}\right)(r+1)^{a}\right)\right. \\
& \left.\quad+\left(\alpha\left(C_{v}^{b *}(\pi)-C_{u}^{b *}(\pi)\right)(r+1)^{a}\right)\right]-\left(d_{u}-d_{v}\right),
\end{aligned}
$$

$L_{u}^{*}\left(\pi^{\prime}\right)-L_{v}^{*}(\pi) \geq 0$, where $p_{u}<p_{v},(a<0)$, and $(\alpha>0)$.

Consequently, $L_{u}^{*}\left(\pi^{\prime}\right) \geq L_{v}^{*}(\pi)$. $\pi^{\prime}$

The total lateness under $\pi$ is strictly less than that under

The maximum tardiness $T_{\max }$ is defined as $T_{\max }=$ $\left\{0, L_{\max }\right\}$. The obtained results can be transferred directly to the case of $1\left|\left[p_{r}+\left(\alpha \times t_{r}^{b}\right)\right](r)^{a}+s_{r}^{* \text { psd }}\right| T_{\max }$.

This completes the proof.

Example 6. Let $p_{1}=90, p_{2}=100, d_{1}=101, d_{2}=102, a=$ -0.322 when learning rate $\% 80, \alpha=0.1, b=0.2$ and $\gamma=0.2$.
Then, the SPT sequence $\pi(S=(1,2))$ leads to $p_{1}^{*}(\pi)=90$, $s_{1}^{*}(\pi)=0, C_{1}^{*}(\pi)=90, p_{2}^{*}(\pi)=80.2, s_{2}^{*}(\pi)=18, C_{2}^{*}(\pi)=$ 188.19 , and $L_{\max }(\pi)=86.19$. Sequence $\pi^{\prime}(S=(2,1))$ leads to $p_{1}^{*}(\pi)=100, s_{1}^{*}(\pi)=0, C_{1}^{*}(\pi)=100, p_{2}^{*}(\pi)=72.2$, $s_{2}^{*}(\pi)=20, C_{2}^{*}(\pi)=192.2$, and $L_{\max }\left(\pi^{\prime}\right)=91.2$.

\section{Common Due Date Problem}

In this section, we tackle common due date problem with past sequence dependent setup times under learning effect and nonlinear deterioration effect. An excellent introduction to common due date problems is given as

$$
1\left|\left[p_{r}+\left(\alpha \times t_{r}\right)\right](r)^{a}+s_{r}^{* \mathrm{psd}}, \quad d_{j}=d\right| \sum\left(E_{j}+T_{j}\right) .
$$

Theorem 7. Consider $1\left|\left[p_{r}+\left(\alpha \times t_{r}\right)\right](r)^{a}+s_{r}^{* p s d}, d_{j}=d\right|$ $\sum\left(E_{j}+T_{j}\right) V$-shape: jobs in set of jobs that are completed on or before the due date are sequenced in nonincreasing order of the $p_{j}$; jobs in set of jobs that are completed after the due date are sequenced in nondecreasing order of the $p_{j}$.

Proof. Consider an optimal schedule $\pi$. Assume, under $\pi$, there are two adjacent jobs, job $J_{u}$, whose sequence is $r$, followed by job $J_{v}$, whose sequence is $r+1$, such that $p_{u}<p_{v}$. $C_{u}^{*}$ and $C_{v}^{*}$ express completion times of $J_{u}$ and $J_{v}$ scheduled at positions $u$ and $v(v=u+1)$ under the nonlinear deterioration and learning effect, respectively. $M$ is starting time of the schedule.

Earliness of job in position $r$ can be calculated by

$$
E_{[r]}=\left(d-C_{r}^{*}\right)
$$

then, from Lemma 1,

$$
\begin{aligned}
E_{u}^{*}(\pi)=d- & {\left[\left[M+p_{1}+\left(\alpha \times M^{b}\right)\right]\right.} \\
& +\sum_{i=2}^{r-1}\left[\left[p_{i}+\left(\alpha \times C_{i-1}^{* b}\right)\right](i)^{a}\right] \\
& +\left[\left[p_{u}+\left(\alpha \times C_{r-1}^{* b}\right)\right](r)^{a}\right] \\
& \left.+\gamma \sum_{i=1}^{r-1} \sum_{j=1}^{i}\left[\left[p_{j}+\left(\alpha \times C_{j-1}^{* b}\right)\right](j)^{a}\right]\right], \\
E_{v}^{*}(\pi)=d- & {\left[\left[M+p_{1}+\left(\alpha \times M^{b}\right)\right]\right.}
\end{aligned}
$$




$$
\begin{aligned}
& +\sum_{i=2}^{r-1}\left[\left[p_{i}+\left(\alpha \times C_{i-1}^{* b}\right)\right](i)^{a}\right] \\
& +\left[\left[p_{u}+\left(\alpha \times C_{r-1}^{* b}\right)\right](r)^{a}\right] \\
& \left.+\gamma \sum_{i=1}^{r-1} \sum_{j=1}^{i}\left[\left[p_{j}+\left(\alpha \times C_{j-1}^{* b}\right)\right](j)^{a}\right]\right] \\
& +\left[\left[\left[\left[p_{v}+\left(\alpha \times C_{u}^{* b}(\pi)\right)\right](r+1)^{a}\right]\right]\right. \\
& +\gamma \sum_{i=1}^{r-1}\left[\left[p_{i}+\left(\alpha \times C_{i-1}^{* b}\right)\right](i)^{a}\right] \\
& \left.+\gamma\left[\left[p_{u}+\left(\alpha \times C_{r-1}^{* b}\right)\right](r)^{a}\right]\right] .
\end{aligned}
$$

If we assume that $t$ is total earliness up to position $r$, then

$$
\begin{aligned}
\sum E^{*}(\pi)=(2 d & -t)-2 \\
\times & {\left[\left[M+p_{1}+\left(\alpha \times M^{b}\right)\right]\right.} \\
& +\sum_{i=2}^{r-1}\left[\left[p_{i}+\left(\alpha \times C_{i-1}^{* b}\right)\right](i)^{a}\right] \\
& +\left[\left[p_{u}+\left(\alpha \times C_{r-1}^{* b}\right)\right](r)^{a}\right] \\
& \left.+\gamma \sum_{i=1}^{r-1} \sum_{j=1}^{i}\left[\left[p_{j}+\left(\alpha \times C_{j-1}^{* b}\right)\right](j)^{a}\right]\right] \\
+ & {\left[\left[\left[\left[p_{v}+\left(\alpha \times C_{u}^{* b}(\pi)\right)\right](r+1)^{a}\right]\right]\right.} \\
& +\gamma \sum_{i=1}^{r-1}\left[\left[p_{i}+\left(\alpha \times C_{i-1}^{* b}\right)\right](i)^{a}\right] \\
& \left.+\gamma\left[\left[p_{u}+\left(\alpha \times C_{r-1}^{* b}\right)\right](r)^{a}\right]\right] .
\end{aligned}
$$

Performing a pairwise interchange on jobs $J_{u}$ and $J_{v}$, we obtain schedule $\pi^{\prime}$. The completion times of the jobs processed before jobs $J_{u}$ and $J_{v}$ are not affected by interchange; then,

$$
\begin{aligned}
E_{v}^{*}\left(\pi^{\prime}\right)=d-[ & {\left[M+p_{1}+\left(\alpha \times M^{b}\right)\right] } \\
& +\sum_{i=2}^{r-1}\left[\left[p_{i}+\left(\alpha \times C_{i-1}^{* b}\right)\right](i)^{a}\right] \\
& +\left[\left[p_{v}+\left(\alpha \times C_{r-1}^{* b}\right)\right](r)^{a}\right]
\end{aligned}
$$$$
\left.+\gamma \sum_{i=1}^{r-1} \sum_{j=1}^{i}\left[\left[p_{j}+\left(\alpha \times C_{j-1}^{* b}\right)\right](j)^{a}\right]\right],
$$$$
E_{u}^{*}\left(\pi^{\prime}\right)=d-\left[\left[M+p_{1}+\left(\alpha \times M^{b}\right)\right]\right.
$$$$
+\sum_{i=2}^{r-1}\left[\left[p_{i}+\left(\alpha \times C_{i-1}^{* b}\right)\right](i)^{a}\right]
$$$$
+\left[\left[p_{v}+\left(\alpha \times C_{r-1}^{* b}\right)\right](r)^{a}\right]
$$$$
\left.+\gamma \sum_{i=1}^{r-1} \sum_{j=1}^{i}\left[\left[p_{j}+\left(\alpha \times C_{j-1}^{* b}\right)\right](j)^{a}\right]\right]
$$$$
+\left[\left[\left[\left[p_{u}+\left(\alpha \times C_{u}^{* b}(\pi)\right)\right](r+1)^{a}\right]\right]\right.
$$$$
+\gamma \sum_{i=1}^{r-1}\left[\left[p_{i}+\left(\alpha \times C_{i-1}^{* b}\right)\right](i)^{a}\right]
$$$$
\left.+\gamma\left[\left[p_{v}+\left(\alpha \times C_{r-1}^{* b}\right)\right](r)^{a}\right]\right] .
$$

If we assume that $t$ is total earliness up to position $r$, then

$$
\begin{aligned}
\sum E^{*}\left(\pi^{\prime}\right)=(2 d & -t)-2 \\
\times & {\left[\left[M+p_{1}+\left(\alpha \times M^{b}\right)\right]\right.} \\
& +\sum_{i=2}^{r-1}\left[\left[p_{i}+\left(\alpha \times C_{i-1}^{* b}\right)\right](i)^{a}\right] \\
& +\left[\left[p_{v}+\left(\alpha \times C_{r-1}^{* b}\right)\right](r)^{a}\right] \\
& \left.+\gamma \sum_{i=1}^{r-1} \sum_{j=1}^{i}\left[\left[p_{j}+\left(\alpha \times C_{j-1}^{* b}\right)\right](j)^{a}\right]\right] \\
+ & {\left[\left[\left[p_{u}+\left(\alpha \times C_{u}^{* b}(\pi)\right)\right](r+1)^{a}\right]\right] } \\
& +\gamma \sum_{i=1}^{r-1}\left[\left[p_{i}+\left(\alpha \times C_{i-1}^{* b}\right)\right](i)^{a}\right] \\
& \left.+\gamma\left[\left[p_{v}+\left(\alpha \times C_{r-1}^{* b}\right)\right](r)^{a}\right]\right]
\end{aligned}
$$


The difference between the values of $\sum E^{*}\left(\pi^{\prime}\right)$ and $\sum E^{*}(\pi)$ is

$$
\begin{aligned}
& \left(\sum E^{*}\left(\pi^{\prime}\right)-\sum E^{*}(\pi)\right) \\
& =\left[\left(\left(p_{v}-p_{u}\right)(r+1)^{a}\right)+\left(\alpha\left(C_{u}^{* b}\left(\pi^{\prime}\right)-C_{v}^{* b}(\pi)\right)(r+1)^{a}\right)\right. \\
& \left.\quad+\left((\gamma+2)\left(p_{u}-p_{v}\right)(r)^{a}\right)\right],
\end{aligned}
$$

$\sum E^{*}\left(\pi^{\prime}\right)-\sum E^{*}(\pi) \leq 0$, where $p_{u}<p_{v},(a<0)$, and $(\alpha, b>$ $0)$.

Consequently, $\sum E^{*}\left(\pi^{\prime}\right) \leq \sum E^{*}(\pi)$; it means that jobs in set of jobs that are completed before the due date are sequenced in nonincreasing order of the $p_{j}$.

If a similar argument follows for the jobs that are started after the common due date $d$, we will obtain the following:

$$
\sum T^{*}\left(\pi^{\prime}\right)-\sum T^{*}(\pi) \geq 0
$$

where $p_{u}<p_{v},(a<0)$, and $(\alpha>0)$.

Consequently, $\sum T^{*}\left(\pi^{\prime}\right) \geq \sum T^{*}(\pi)$; it means that jobs in set of jobs that are completed after the due date are sequenced in nondecreasing order of the $p_{j}$.

\section{Conclusions}

In this paper, we introduced some problems against due dates with past sequence dependent setup times under learning effect and nonlinear deterioration effect. In this study, effects of learning and deterioration are considered simultaneously. We present that some problems remain polynomially solvable. These problems are minimizing total lateness, total tardiness, maximum lateness (with agreeable due dates), maximum tardiness (with agreeable due dates), and earliness/tardiness problem with common due date.

\section{Conflict of Interests}

The author declares that there is no conflict of interests regarding the publication of this paper.

\section{References}

[1] T. C. E. Cheng, C. Wu, and W. Lee, "Some scheduling problems with deteriorating jobs and learning effects," Computers and Industrial Engineering, vol. 54, no. 4, pp. 972-982, 2008.

[2] Y. Yin, D. Xu, and J. Wang, "Some single-machine scheduling problems with past-sequence-dependent setup times and a general learning effect," International Journal of Advanced Manufacturing Technology, vol. 48, no. 9-12, pp. 1123-1132, 2010.

[3] G. Mosheiov, "Scheduling problems with a learning effect," European Journal of Operational Research, vol. 132, no. 3, pp. 687-693, 2001.

[4] D. Biskup, "Single-machine scheduling with learning considerations," European Journal of Operational Research, vol. 115, no. 1, pp. 173-178, 1999.

[5] G. Mosheiov and J. B. Sidney, "Scheduling with general jobdependent learning curves," European Journal of Operational Research, vol. 147, no. 3, pp. 665-670, 2003.
[6] G. Mosheiov, "A-shaped policies to schedule deteriorating jobs," The Journal of the Operational Research Society, vol. 47, no. 9, pp. 1184-1191, 1996.

[7] J. N. D. Gupta and S. K. Gupta, "Single facility scheduling with nonlinear processing times," Computers and Industrial Engineering, vol. 14, no. 4, pp. 387-393, 1988.

[8] A. S. Kunnathur and S. K. Gupta, "Minimizing the makespan with late start penalties added to processing times in a single facility scheduling problem," European Journal of Operational Research, vol. 47, no. 1, pp. 56-64, 1990.

[9] B. Alidaee and N. K. Womer, "Scheduling with time dependent processing times: review and extensions," The Journal of the Operational Research Society, vol. 50, no. 7, pp. 711-720, 1999.

[10] X. Wang and T. C. E. Cheng, "Single-machine scheduling with deteriorating jobs and learning effects to minimize the makespan," European Journal of Operational Research, vol. 178, no. 1, pp. 57-70, 2007.

[11] J. Wang, "Single-machine scheduling problems with the effects of learning and deterioration," Omega, vol. 35 , no. 4, pp. 397402, 2007.

[12] M. D. Toksari and E. Güner, "Minimizing the earliness/tardiness costs on parallel machine with learning effects and deteriorating jobs: A mixed nonlinear integer programming approach," International Journal of Advanced Manufacturing Technology, vol. 38, no. 7-8, pp. 801-808, 2008.

[13] M. D. Toksari and E. Güner, "Parallel machine earliness/tardiness scheduling problem under the effects of position based learning and linear/nonlinear deterioration," Computers and Operations Research, vol. 36, no. 8, pp. 2394-2417, 2009.

[14] E. Güner and M. D. Toksari, "Single machine scheduling problems against due date with the past sequence dependent setup times under learning and deterioration effects," in Proceedings of the 38th International Conference on Computers and Industrial Engineering, vol. 38, pp. 1923-1930, Beijing, China, 2008.

[15] C. Koulamas and G. J. Kyparisis, "Single-machine scheduling problems with past-sequence-dependent setup times," European Journal of Operational Research, vol. 187, no. 3, pp. 1045-1049, 2008.

[16] D. Biskup and J. Herrmann, "Single-machine scheduling against due dates with past-sequence-dependent setup times," European Journal of Operational Research, vol. 191, no. 2, pp. 587-592, 2008. 


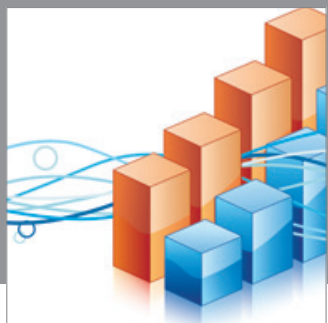

Advances in

Operations Research

mansans

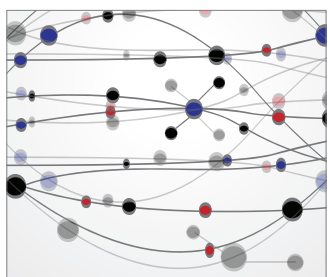

The Scientific World Journal
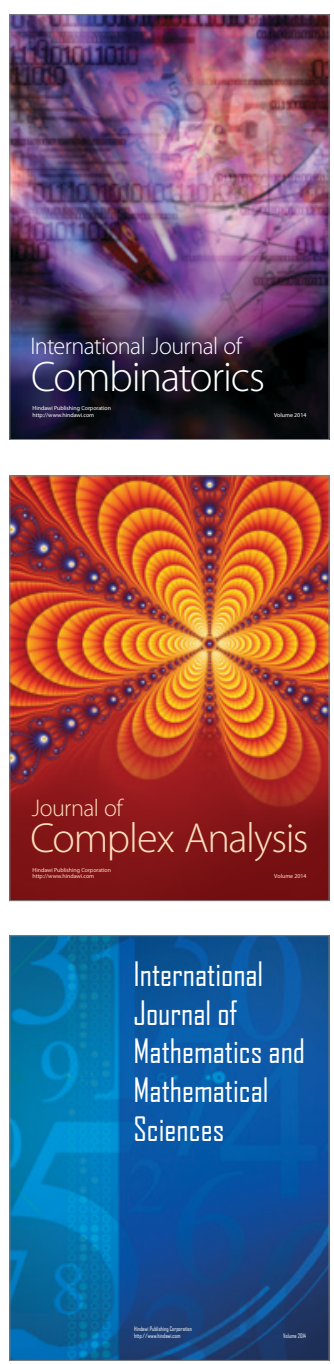
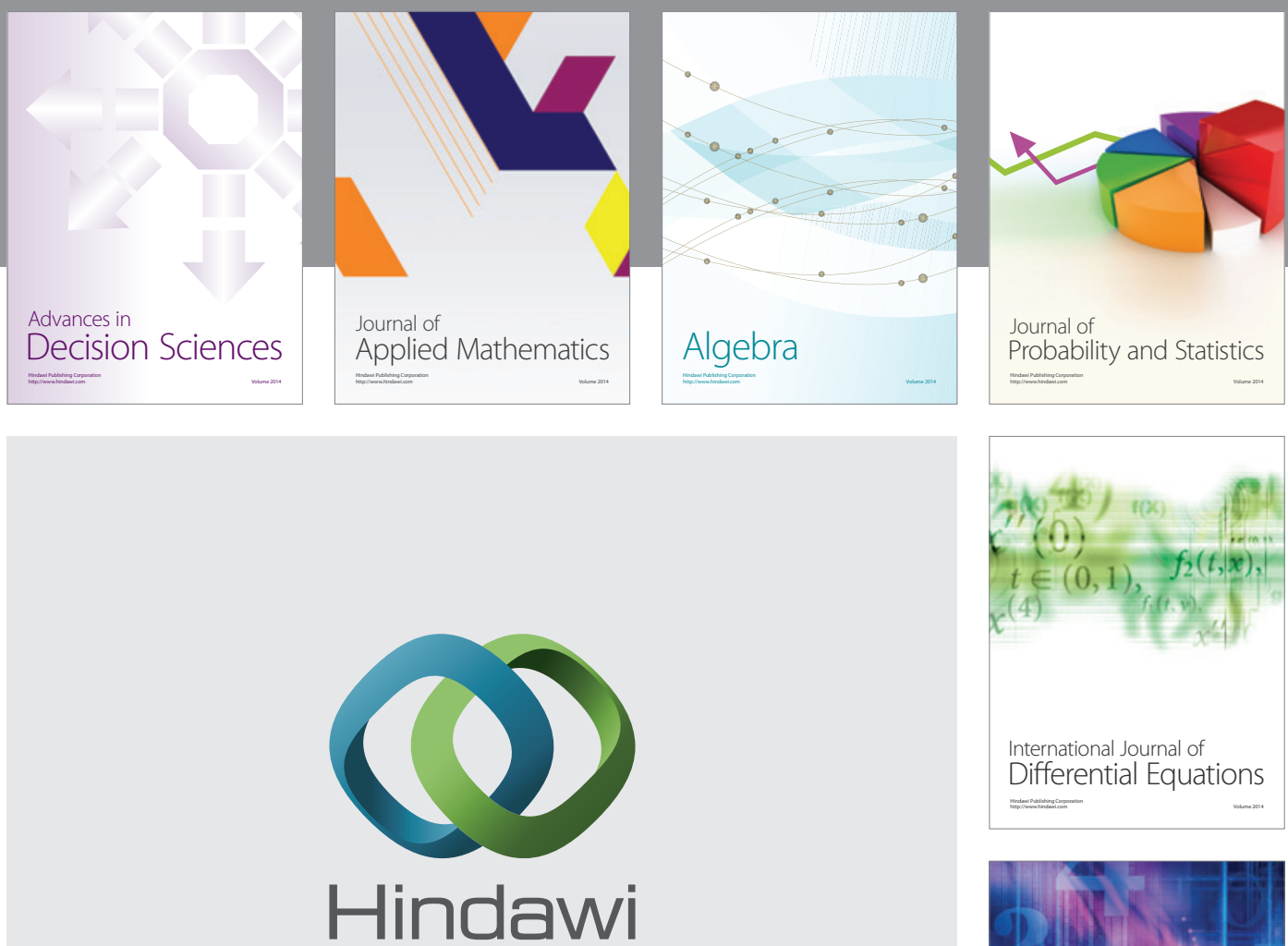

Submit your manuscripts at http://www.hindawi.com
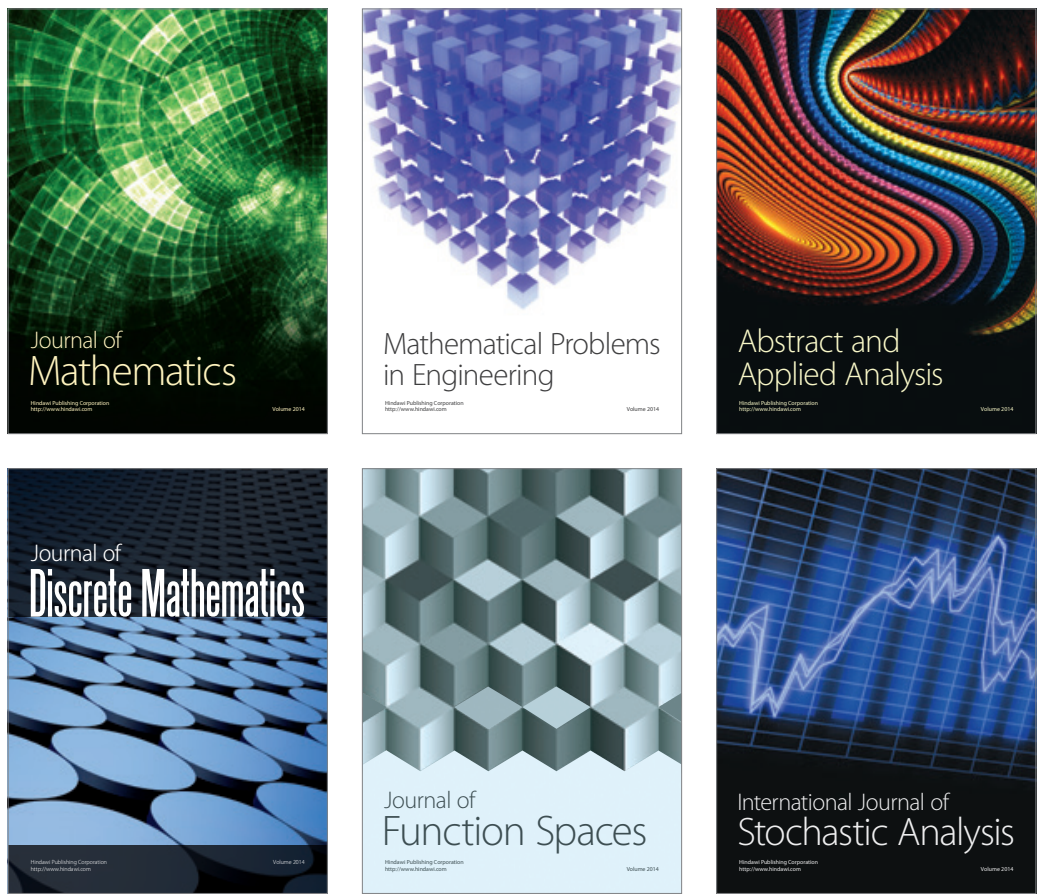

Journal of

Function Spaces

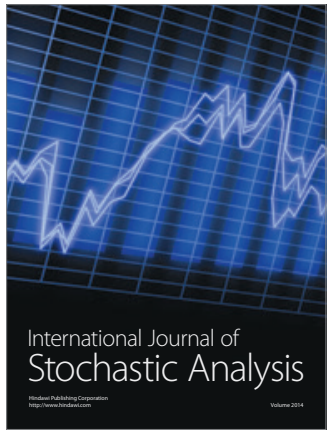

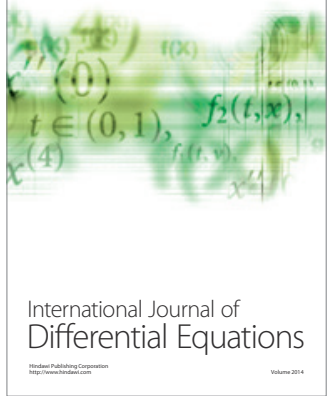
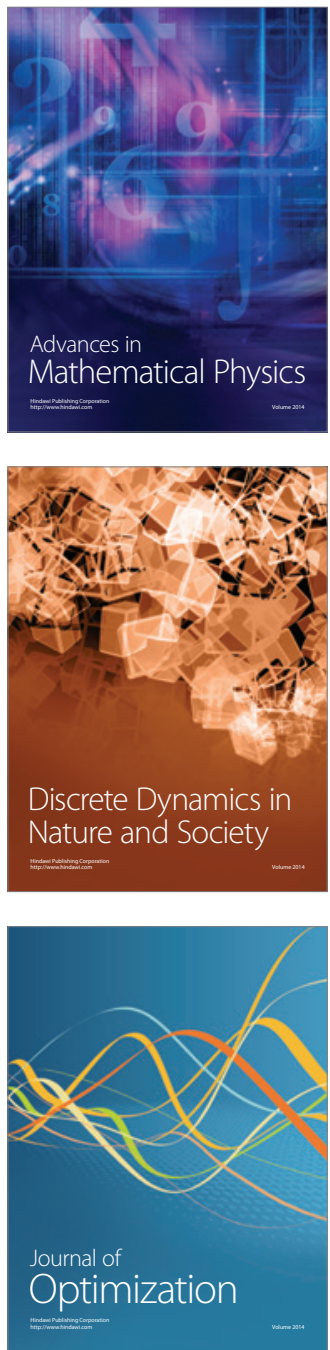